\title{
DNA Words Based on an Enhanced Algorithm of Multi-objective Particle Swarm Optimization in a Continuous Search Space
}

\author{
Krishna Veni Selvan, Mohd Saufee Muhammad and \\ Sharifah Masniah Wan Masra \\ Department of Electronics Engineering \\ Faculty of Engineering \\ Universiti Malaysia Sarawak \\ 94300 Kota Samarahan, Sarawak, Malaysia \\ kveni52@yahoo.com,msaufee@feng.unimas.my, \\ wmmasnia@feng.unimas.my
}

\begin{abstract}
In this paper, particle swarm optimization algorithm in a continuous search space is implemented to generate a set of DNA words. A single swarm with 20 particles is used to find the best solution (gbest). Here, each particle has a number of sub-particles which is referred as the number of sequences to be designed. Overall, the particle which has the optimum fitness value is considered as the best solution. Fitness value of a particle is computed from the average value of all the applied objective functions. The solution obtained from this algorithm is found to be better as compared with other approaches. Furthermore, it has a fast convergence towards the optimum fitness value.
\end{abstract}

Keywords - DNA molecules; DNA words; fitness; continuous particle swarm optimization (CPSO)

\section{INTRODUCTION}

According to Adleman [1], who pioneered a research work on molecular computing to solve Hamiltonian path problem in 1994, the achievement of molecular computing highly relies on the DNA strands used during its in vitro experiments. This motivated and contributed to a wide range of idea in designing DNA sequences. However, the designed oligonucleotides ought to minimize several design criteria or objectives concurrently. Therefore, DNA words designing is a complex multi-objective constrained optimization assignment. In 1995, Eberhart and Kennedy [2], [3] introduced a well known optimization technique called particle swarm optimization (PSO). The technique was inspired by the nature concepts of bird flocking and fish schooling. PSO is a great optimization approach. In this paper, an enhanced continuous particle swarm optimization (CPSO) algorithm is proposed for generating the DNA sequences.

\author{
Zuwairie Ibrahim and Kian Sheng Lim \\ Center for Artificial Intelligence and Robotics (CAIRO) \\ Department of Mechatronics and Robotics \\ Faculty of Electrical Engineering \\ Universiti Teknologi Malaysia \\ 81310 Skudai, Johor, Malaysia \\ zuwairie@fke.utm.my, kiansheng860524@yahoo.com
}

\section{DESIGN REQUIREMENTS}

Some combinatorial constraints act as the main role in designing a good series of DNA molecules for molecular computation. The designed sequences should be as unique as possible, thermodynamically stable, non-self hybridized, non-cross hybridized with others and have good chemical properties. In this research, four objective functions and two constraints are being considered. The objective functions are H-measure, similarity, hairpin and continuity, while the two constraints are melting temperature and GC-content. A good set of designed DNA oligonucleotides must optimize all the aforementioned functions simultaneously.

H-measure calculates the possible number of hybridizing molecules among all the designed sequences in an anti-parallel direction. Similarity calculates the number of identical molecules between other sequences in a parallel direction. Hairpin computes the possibility of self hybridization of a DNA string. Continuity calculates the occurrence of similar molecules in a strand as it weakens the strands stability. The calculations of the objective functions are in accordance with Shin et al. [4] and several parameter values, as exhibited in Table I. 